\title{
COMMENTARY
}

\section{A healthy dose of scepticism: Four good reasons to think again about protective effects of alcohol on coronary heart disease}

TANYA CHIKRITZHS', KAYE FILLMORE ${ }^{2} \&$ TIM STOCKWELL ${ }^{3}$

${ }_{1}$ National Drug Research Institute, Curtin University of Technology, Perth, Australia, 2 Department of

Social and Behavioral Sciences, University of California, San Francisco, USA, and ${ }_{3}$ Center for

Addictions Research of British Columbia, University of Victoria,Victoria BC, Canada

\section{Introduction}

There was once a time when sceptics of the purported beneficial effect of moderate alcohol consumption on coronary heart disease (CHD) were relegated to the ranks of 'doubters of manned lunar missions and members of the Flat Earth Society' [1] (p.116); that time has now past. Today, epidemiological evidence for an apparent protective effect of alcohol on CHD is being challenged; the following is a synopsis of those various challenges as they currently stand.

\section{The challenges}

Scientific evidence for a potential cardio-protective effect of moderate alcohol consumption first entered mainstream alcohol research literature in the mid 1970s in the form of a ' $J$-shaped curve' - as it is now commonly referred to. In a general sense, the J-curve describes a relationship between a risk factor and a disease which does not proceed in the typical linear manner but where, at some point, the relationship 'changes from negative to positive' [2] (p.824). For alcohol and all-cause mortality, the J-curve corresponds to a situation in which teetotallers fare worse than moderate drinkers who in turn fare better than heavier drinkers (see Figure 1 for example). Buoyed by plausible biological mechanisms demonstrated in laboratory settings, (e.g. increased profile of 'good' fats and thrombolytic effects) the rapid proliferation of evidence supporting the notion of a J-shaped curve for alcohol and CHD has been enthusiastically received and continues to hold the interest of researchers, health professionals and the general public.

Encouraged, at least in part, by the apparent protective effect of alcohol on CHD, the pursuit of evidence for potential protective effects of alcohol on other disease states is a popular focus of many epidemiologists nowadays; particularly for major causes of mortality and morbidity such as type II diabetes, dementia and rheumatoid arthritis (see Figure 1).

\section{[INSERT FIGURE 1 HERE]}

Misclassification error. An alternative explanation for the apparent protective effect of alcohol on CHD was proposed by Shaper, Wannamethee and Walker (1988) involving the systematic misclassification of ex-drinkers and occasional drinkers as 'abstainers' in longitudinal studies resulting thus negatively biasing the health status of the reference group of abstainers [3]. After the publication of many subsequent studies purporting to have ruled-out these misclassification errors, the argument was relegated, by most, to history - until very recently. After reviewing, in detail, the questions put to respondents regarding alcohol use among 54 all-cause mortality studies and 35 CHDspecific morality studies, Fillmore et al. found only 7 of the former and 2 of the latter to 
be without misclassification error. All of the studies affected by misclassification error showed protective effects, and none of the few which were error-free displayed a protective effect [4]. The issue of misclassification error has since been the subject of serious debate in the scientific literature (see commentaries on [4] at http://www.informaworld.com/smpp/title $\sim$ content=g773385479 $\mathrm{db}=\mathrm{all}$ ) and new research has begun to conscientiously re-assess the contribution of misclassification error to apparent protective effects for alcohol. A recently published Australian study found that when misclassification error was accounted for, there was no apparent protective effect of usual daily alcohol consumption for males. There was a protective effect for females, but this was limited to wine consumption only [5]. This was consistent with a re-analysis of [4] which found that the protective effect disappeared for men but not women when abstainer biases were controlled [6].

Confounding. To our knowledge, there has never been a randomised controlled trial which has examined whether alcohol consumption reduces the likelihood of death from CHD. Such a study would be difficult to undertake because of ethical and practical concerns. Studies which have produced J-shaped curves for alcohol and disease have been dominated by prospective cohort studies [7]. Due to the very nature of these population-based studies, it is practically impossible to completely rule-out the influence of uncontrolled confounding on results. Jackson et al. offered a timely reminder of the pit-falls awaiting premature attribution of causality when they recalled the once widely held view that hormone replacement therapy offered post-menopausal women protection against CHD - a view which was ultimately proved, by randomised controlled trial, to have been based on artefactual evidence drawn from cohort studies [8]. It is well to remember that, for the majority of economically developed populations (i.e. U.S. and European countries) for which epidemiological studies have demonstrated J-shaped curves, people who abstain from drinking are in themselves, atypical. With regard to alcohol, among these populations, about $90 \%$ of all risk factors for CHD are more likely to occur among non-drinkers than moderate drinkers. Abstainers are typically: older; poorer; less educated; in worse health; and have lower levels of medical well-being, access to health care or prevention health services than moderate drinkers [9]. In an attempt to address potential confounding of this sort - at least in part - some studies have excluded participants on the basis of known current and past health history (e.g. [10]).

Unfortunately, the exclusion of participants on the basis of current and past ill-health has the regrettable side-effect of severely reducing sample size. In the 2006 Mukamal et al. study for example, a baseline sample of over 50,000 respondents was reduced to slightly less than 9,000 after excluding those with cardiovascular disease, cancer, diabetes and unhealthy lifestyles [10]. However, Fillmore et al. estimated that had Mukamal et al. also excluded respondents diagnosed with hypertension, hypercholesterolemia, gall bladder disease, arthritis, bronchitis, asthma, medication use, gout and poor or fair health (assuming all mutually exclusive), eligible participants would have numbered only 407 - which is to be expected, given the average age at entry for most CHD studies [7].

Moreover, using simulated models, Marschner et al. have demonstrated how the exclusion of participants on the basis of pre-existing illness may artificially create a spurious J-shaped curve when the underlying relationship between alcohol and disease is fundamentally linear [11]. Like the arguments regarding misclassification error, the 
case for confounding effects and their influence on apparent protective effects of alcohol remains unresolved.

Self-report, recall bias and drinker 'drift'. Most epidemiological studies rely heavily on the ability of respondents to accurately report their own current and past alcohol consumption. Yet it is well documented that most self-report drinking surveys underreport consumption (e.g. [12]). In relation to studies of protective effects of alcohol and CHD, it is particularly important that participants self-reporting as 'abstainers' have been able to accurately identify and define their own life-time drinking status. Caldwell et al. examined the drinking histories of self-identified abstainers and occasional drinkers. Respondents were asked about their current alcohol consumption at ages 16, 23, 33, 42 and 45 (e.g. non-drinker, occasional drinker, drink on most days etc). More than $60 \%$ of respondents who claimed to be 'never' drinkers at age 45 -years had actually reported drinking alcohol at any one of the previous follow-up surveys. Nearly a quarter of self-reported 45-year-old 'never' drinkers had previously reported drinking at least once a week, and more than a third had consumed alcohol more than occasionally. Some $56 \%$ of 45 -year-olds self-reported to be 'occasional only' drinkers had previously reported drinking at least once a week [13]. It would appear, at least for this population, that true life-time abstainers are even rarer than first thought.

Related to this, is of course the inconvenient fact that people do not maintain one single drinking level or style throughout life - abstainers, for example, often drift between drinking categories (and non-drinking) over time [14]. It is very difficult, if not impossible, for epidemiological studies to 'capture' respondents who have stable lifetime drinking patterns or who can be considered reliable exemplars of a certain 'type' of drinker (e.g. moderate drinker, risky drinker, abstainer).

Drinking patterns. There is limited evidence that the relationship between alcohol and some disease incidence is a function of the manner or pattern in which it is consumed (e.g. regular consumption vs. occasional drinking to intoxication). A review by Rehm and colleagues concluded that hypertension and sudden death from cardiovascular disease may be related to occasions of heavy drinking and intoxication [15]. Along similar lines, others have suggested that conclusions regarding alcohol's cardioprotective effects have been based on pooled observations dominated by studies of Western populations where drinking patterns can be 'characterised by largely moderate and regular pattern of alcohol consumption’ [16, p.330]. Nonetheless, proportionately, very few epidemiological studies in this domain have addressed the complex issue of drinking pattern (most are only able to provide crude measures over limited time periods) and apparent protective effects of alcohol consumption on CHD. One Australian case-control study failed to find a protective effect for coronary events among 'binge' [sic] drinkers compared to abstainers but did find a protective effect for regular moderate drinkers compared to abstainers [17].

\section{Conclusion}

The study of disease at population levels is a challenging science for which the attribution of causality is a laborious and painstaking process requiring many years of collective scientific endeavour. The brief summary of evidence presented does not allow a conclusive answer to the question of whether or not alcohol protects against death or morbidity from CHD. Indeed, substantial evidence from laboratory studies suggests that, in a physiological sense, the existence of such an effect is entirely 
plausible. There is nevertheless, growing evidence to suggest that the magnitude of any cardio-protective effect has been exaggerated and that the science of epidemiology is yet to conclusively mitigate doubts regarding significant cardio-protective effects at a population level. We further argue that, sufficient weight of evidence has accrued for the wider research, health and medical community to think again about our collective acceptance of alcohol as a remedy for one the most common causes of death facing the developed world.

The implications of the arguments presented here extend beyond the relationship between alcohol and CHD. With differing degrees of scientific veracity, alcohol has been implicated in both the popular and scientific press as having a protective effect for at least a dozen conditions other than CHD, including: ischaemic stroke; gall stones; dementia; type II diabetes; helicobacter pylori infection; rheumatoid arthritis; prostate cancer; kidney stones; osteoporosis; melanoma; leukaemia; the common cold; and hearing loss (see. http://en.wikipedia.org/wiki/Alcohol_consumption_and_health as an example of popular press). The concern is that the same methodological issues identified for alcohol and CHD may also unduly influence apparent findings that alcohol protects against mortality and morbidity for these other disease states. Yet, for societies that have a strong appetite for new health and medical developments, propelled by an increasingly accessible media, it is inevitable that when repeated and recounted, even tenuous, briefly-communicated findings can come to be treated as scientific fact.

\section{References}

[1] Stockwell T. Alcohol and cardiovascular disease: Still a research priority? Med J Aust. 2000;173:116-117.

[2] Marschner I, Simes J, Keech A. Biases in the identification of risk factor thresholds and J-curves. Am J Epidemiol. 2007;21:824-831.

[3] Shaper G, Wannamethee G, Walker M. (1988) Alcohol and mortality in British men: Explaining the U-shaped curve. Lancet 1988; 2: 1267

[4] Fillmore K, Stockwell T, Kerr W, et al. Moderate alcohol use and reduced mortality risk: systematic error in prospective studies. Addiction Research and Theory 2006;14(2):101-132.

[5] Harriss L, English D, Hopper J. et al. Alcohol consumption and cardiovascular mortality accounting for possible misclassification of intake: 11-year follow-up of the Melbourne Collaborative Cohort Study. Addiction 2007;102:1574-1585.

[6] Stockwell T, Chikritzhs T, Bostrom A. et al. Lives lost and saved from alcohol consumption in Australia and Canada: Scenario analyses using different assumptions about purported health benefits. J Stud Alcohol 2007;68(3):345-352.

[7] Fillmore K, Stockwell T, Chikritzhs, T, et al. Moderate alcohol use and reduced mortality risk: Systematic error in prospective studies and new hypotheses. Ann Epidemiol. 2007;17:S16-S23. 
[8] Jackson R, Broad J, Connor J, Wells S. Alcohol and ischaemic heart disease: probably no free lunch. Lancet 2005;366(3):1911.

[9] Naimi T, Brown D, Brewer R, et al. Cardiovascular risk factors and confounders among nondrinking and moderate-drinking U.S. adults. American Journal of Preventative Medicine 2005;28:369-73.

[10] Mukamal K, Chiuve S, Rimm E. Alcohol consumption and risk for coronary heart disease in men with healthy lifestyles. Arch Intern Med. 2006;166:2145-2150.

[11] Marschner I, Simes J. Keech A. Biases in the identification of risk factor thresholds and J-curves. Am J Epidemiol. 2007;21:824-831.

[12] Dawson D. Alcohol consumption, alcohol dependence and all-cause mortality. Alcohol Clin Exp Res. 2000;24:72-81.

[13] Caldwell T, Rodgers B, Power C, et al. Drinking histories of self-identified lifetime abstainers and occasional drinkers: Findings from the 1958 British Birth Cohort study. Alcohol Alcohol 2006;41(6);650-654.

[14] Kerr W, Fillmore K, Bostrom A. Stability of alcohol consumption over time: evidence from three longitudinal surveys from the United States. J Stud Alcohol 2002;63:325-333.

[15] Rehm J, Room R, Monteiro M, et al. Alcohol use. In: Ezzati M, Lopez, A, Rodgers A, Murray, C, editors. Comparative quantification of health risks. Global and regional burden of disease attributable to selected major risk factors. Volume 1. Geneva: World Health Organization;2004. p.959-1108.

[16] Britton A, McKee M. The relation between alcohol and cardiovascular disease in Eastern Europe: explaining the paradox. J. Epidemiol Community Health; 2000;54: 328-332.

[17] McElduff P, Dobson A. How much alcohol and how often? Population based case-control study of alcohol consumption and risk of a major coronary event. BMJ 1997;314:1159. 